\title{
PROPERTIES OF NARROW CORONAL MASS EJECTIONS OBSERVED WITH LASCO
}

\author{
S. Yashiro, ${ }^{1,2}$ N. Gopalswamy, ${ }^{2}$ G. Michałek, ${ }^{1,2,3}$ and R. A. Howard ${ }^{4}$ \\ ${ }^{1}$ The Catholic University of America, Washington, DC 20064, USA \\ ${ }^{2}$ NASA Goddard Space Flight Center, Greenbelt, MD 207r1, USA \\ ${ }^{3}$ Astronomical Observatory of Jagiellonian University, Cracow, Poland \\ ${ }^{4}$ Naval Research Laboratory, Washington DC 20375, USA
}

\begin{abstract}
We report the statistical properties of narrow coronal mass ejections (CMEs, angular width $<20^{\circ}$ ) with particular emphasis on comparison with normal CMEs. We investigated 806 narrow CMEs from an online LASCO/CME catalog and found that (1) the fraction of narrow CMEs increases from $12 \%$ to $22 \%$ towards solar maximum, (2) during the solar maximum, the narrow CMEs are generally faster than normal ones, (3) the maximum speed of narrow CMEs $\left(1141 \mathrm{~km} \mathrm{~s}^{-1}\right)$ is much smaller than that of the normal CMEs (2604 $\left.\mathrm{km} \mathrm{s}^{-1}\right)$. These results imply that narrow CMEs do not form a subset of normal CMEs and have a different acceleration mechanism from normal CMEs.
\end{abstract}

\section{INTRODUCTION}

From January 1996 to December 2001, more than 5000 coronal mass ejections (CMEs) have been observed by the Large Angle and Spectrometric Coronagraph (LASCO) on board Solar and Heliospheric Observatory $(\mathrm{SOHO})$. The median of apparent angular width in the LASCO C2 field of view is $50^{\circ}$ (St. Cyr et al., 2000), but angular width is widely distributed. CMEs with maximum apparent width of $360^{\circ}$ are known as "halo" CMEs which are actively investigated for space weather applications (Gopalswamy et al. 2000, St. Cyr et al. 2000, Webb et al. 2000). It has been thought that only fast and wide CMEs have an important role in production of large solar energetic particles (SEPs, see e.g., Gopalswamy et al. 2002). Kahler et al. (2001) reported that some impulsive SEP events were also associated with fast but narrow CMEs. Narrow CMEs also may have an important role in impulsive SEP production or propagation to the interplanetary medium, therefore it is important to understand the characteristics and origin of narrow CMEs.

Normal CMEs are likely to have the well known three-part structure and they can be explained as due to the expansion of flux tubes. In contrast, the narrow CMEs seem to be mass flows in vertical flux tubes. Wang et al. (1998) investigated the solar surface counterparts of 27 jet-like CMEs observed above the polar coronal holes (width $\sim 3^{\circ}-7^{\circ}$ ) using the EUV Imaging Telescope (EIT) on board $S O H O$, and found that these particular type of narrow CMEs were the outward extensions of EUV jets (see also, Wang and Sheeley, 2002). They also found that the leading edges of jet-like CMEs propagate at speeds of $400-1100 \mathrm{~km} \mathrm{~s}^{-1}$, while the bulk of their material travels at around $250 \mathrm{~km} \mathrm{~s}^{-1}$. Gilbert et al. (2001) examined properties of 15 narrow CMEs and found that apparent speeds ranged from $159-630 \mathrm{~km} \mathrm{~s}^{-1}$. They also investigated their surface associations and found that most narrow CMEs originate near a relatively sharp bend in a polarity-reversal line. They concluded that there is no obvious difference between the narrow and normal CMEs other than their appearance. However, little is known of the statistical properties of narrow CMEs and the difference with those of normal CMEs. In this paper, we present the statistical properties (angular width, speed, and location) of narrow CMEs to compare them with those of normal CMEs using an online LASCO/CME catalog (http://cdaw.gsfc.nasa.gov/CME_list/). 
In order to identify CMEs, we examined LASCO running difference movies on the computer monitor. We defined all transient moving features, for which height-time measurements can be made as CMEs. The online CME catalog contains almost all major CMEs detected by the LASCO $\mathrm{C} 2$ and $\mathrm{C} 3$ coronagraphs. However, we have missed many very faint and narrow CMEs, since it is difficult to identify them. For example, almost all 27 jet-like CMEs examined by Wang et al. (1998) are not listed in the CME catalog since they are vary faint and can be seen in only $1-3$ LASCO images. We should say that we examined a subset of narrow CMEs.

\section{WIDTH DISTRIBUTION}

Figure 1 shows distributions of apparent angular width from 1996 to 2001. Last bins (>180) include halo CMEs. During solar minimum (1996 - 1997), the shape of distributions was simple with a peak of $\sim 40^{\circ}$. During 1998 - 2000, bi-modal distributions were found with peaks at $\sim 15^{\circ}$ and $\sim 50^{\circ}$. In 2001, the bi-modal distribution disappeared. From the distributions during the solar maximum, we defined the narrow CMEs as those with angular width less than $20^{\circ}$ (light bars in Fig. 1); CMEs with angular width greater than $20^{\circ}$ are defined as normal CMEs (dark bars in Fig. 1). Note that this definition is different from the previous studies. Gilbert et al. (2001) used a critical width of $15^{\circ}$ and Wang et al. (1998) examined jet-like CMEs with angular width of $3^{\circ}-7^{\circ}$. The number ratio of narrow and normal CMEs was $12 \%$ (57/474) during solar minimum (1996 - 1997) and 22\% (440/2041) during solar maximum (1999 - 2000). If a narrow CME occurred just after a halo CME, it is quite difficult to identify the narrow CME. Therefore, the number ratio during solar maximum could be much higher.
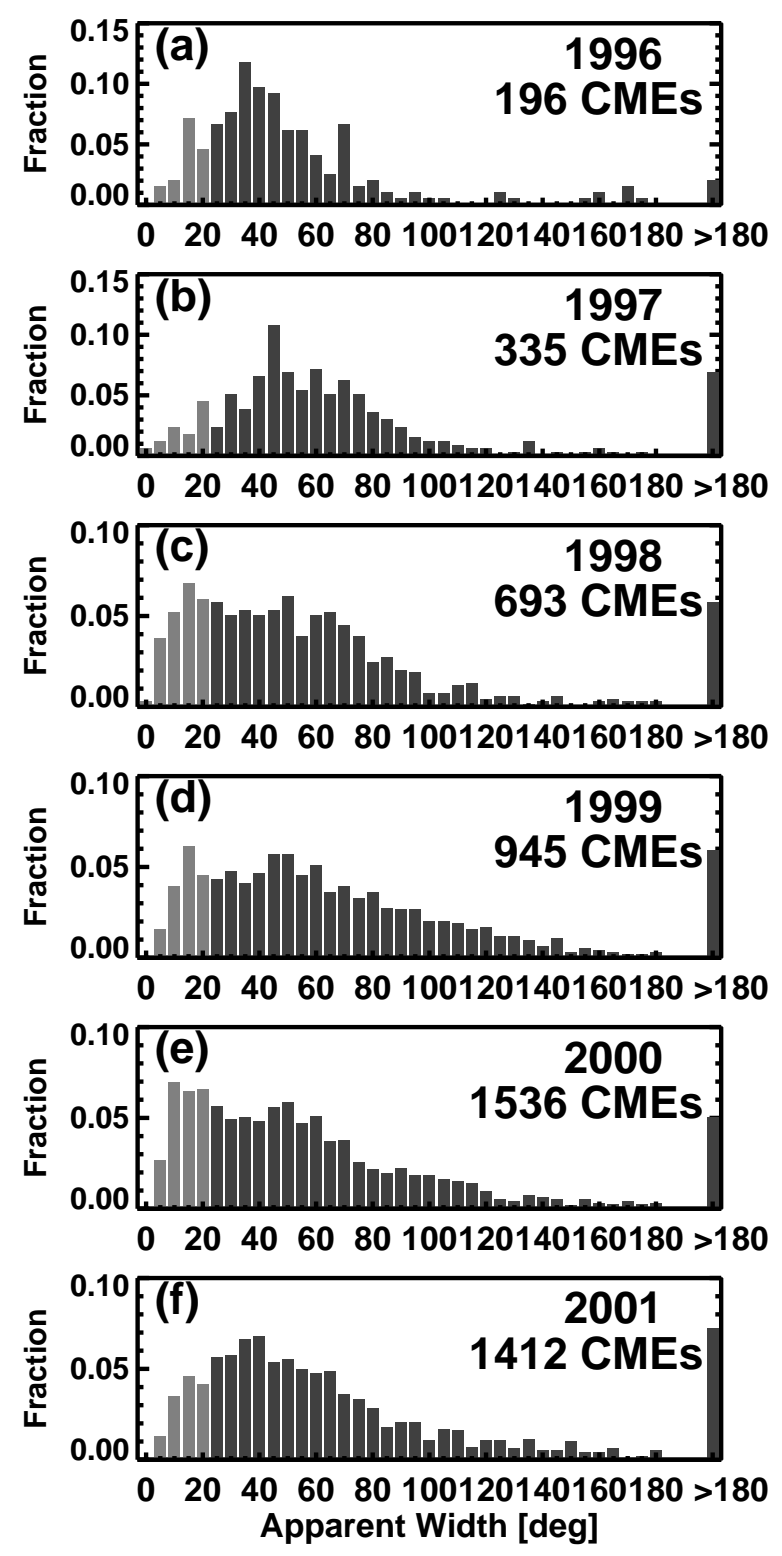

Fig. 1. Width distributions of narrow (lightshaded) and normal (dark-shaded) CMEs from 1996 to 2001.

\section{SPEED DISTRIBUTION}

We measured the heliocentric distance of the leading edge of CMEs in each LASCO image, and obtained CME's height as a function of time. The height-time plots are then fitted to first order polynomials to characterize the motion of the CMEs. The first order fit gives an average speed within the LASCO field of view. We used this average speed even if the second order fit was more suitable.

Table 1 summarizes the annual variation of median (average) speeds for narrow and normal CMEs. Solar cycle variation is clearly seen. The median (average) speeds during solar minimum $(1996-1997)$ were 237 (269) $\mathrm{km} \mathrm{s}^{-1}$ for narrow CMEs and $257(299) \mathrm{km} \mathrm{s}^{-1}$ for normal CMEs. Though the data sample was not large, the median speed of narrow CMEs was slightly lower than that of normal CMEs during solar minimum. In contrast, during solar maximum $(1999$ - 2000), the median (average) speeds were 527 (545) $\mathrm{km} \mathrm{s}^{-1}$ for narrow CMEs and $429(491) \mathrm{km} \mathrm{s}^{-1}$ for normal CMEs. Therefore, the median speed of narrow CMEs was higher than that of normal CMEs. 
Table 1. Speeds of Narrow and Normal CMEs

\begin{tabular}{lll}
\hline Year & $\begin{array}{l}\text { Narrow CME } \\
\text { Median (Average) }\end{array}$ & $\begin{array}{l}\text { Normal CME } \\
\text { Median (Average) }\end{array}$ \\
\hline 1996 & $255(270) \mathrm{km} \mathrm{s}^{-1}$ & $237(269) \mathrm{km} \mathrm{s}^{-1}$ \\
1997 & $220(268) \mathrm{km} \mathrm{s}^{-1}$ & $268(315) \mathrm{km} \mathrm{s}^{-1}$ \\
1998 & $366(390) \mathrm{km} \mathrm{s}^{-1}$ & $361(423) \mathrm{km} \mathrm{s}^{-1}$ \\
1999 & $559(584) \mathrm{km} \mathrm{s}^{-1}$ & $422(488) \mathrm{km} \mathrm{s}^{-1}$ \\
2000 & $496(528) \mathrm{km} \mathrm{s}^{-1}$ & $434(495) \mathrm{km} \mathrm{s}^{-1}$ \\
2001 & $442(476) \mathrm{km} \mathrm{s}^{-1}$ & $393(475) \mathrm{km} \mathrm{s}^{-1}$ \\
\hline
\end{tabular}

Figure 2 shows the speed distributions from 1996 to 2001 for narrow (light bars) and normal (dark bars) CMEs. The fractions in $100 \mathrm{~km} \mathrm{~s}^{-1}$ interval are plotted. We can clearly see that the speeds of narrow and normal CMEs increased towards solar maximum. For some CMEs, we could not measure the CME speeds, therefore the number of events decreased compared to that used in the width distribution.

During solar minimum, it is difficult to find any difference between the shapes of narrow and normal distributions because of the small sample size for narrow CMEs. In contrast, during solar maximum, several differences can be seen. (1) The speed distribution of narrow CMEs had a peak of $400-500$ $\mathrm{km} \mathrm{s}^{-1}$, while $300-400 \mathrm{~km} \mathrm{~s}^{-1}$ for normal CMEs. (2) The population of narrow CMEs sharply declined at higher speeds, while the distribution of normal CMEs had a high speed tail. No narrow CMEs with speed higher than $1200 \mathrm{~km} \mathrm{~s}^{-1}$ was observed. The maximum speed of narrow CMEs $\left(1141 \mathrm{~km} \mathrm{~s}^{-1}\right)$ was much lower than that of normal CMEs $\left(2600 \mathrm{~km} \mathrm{~s}^{-1}\right)$.
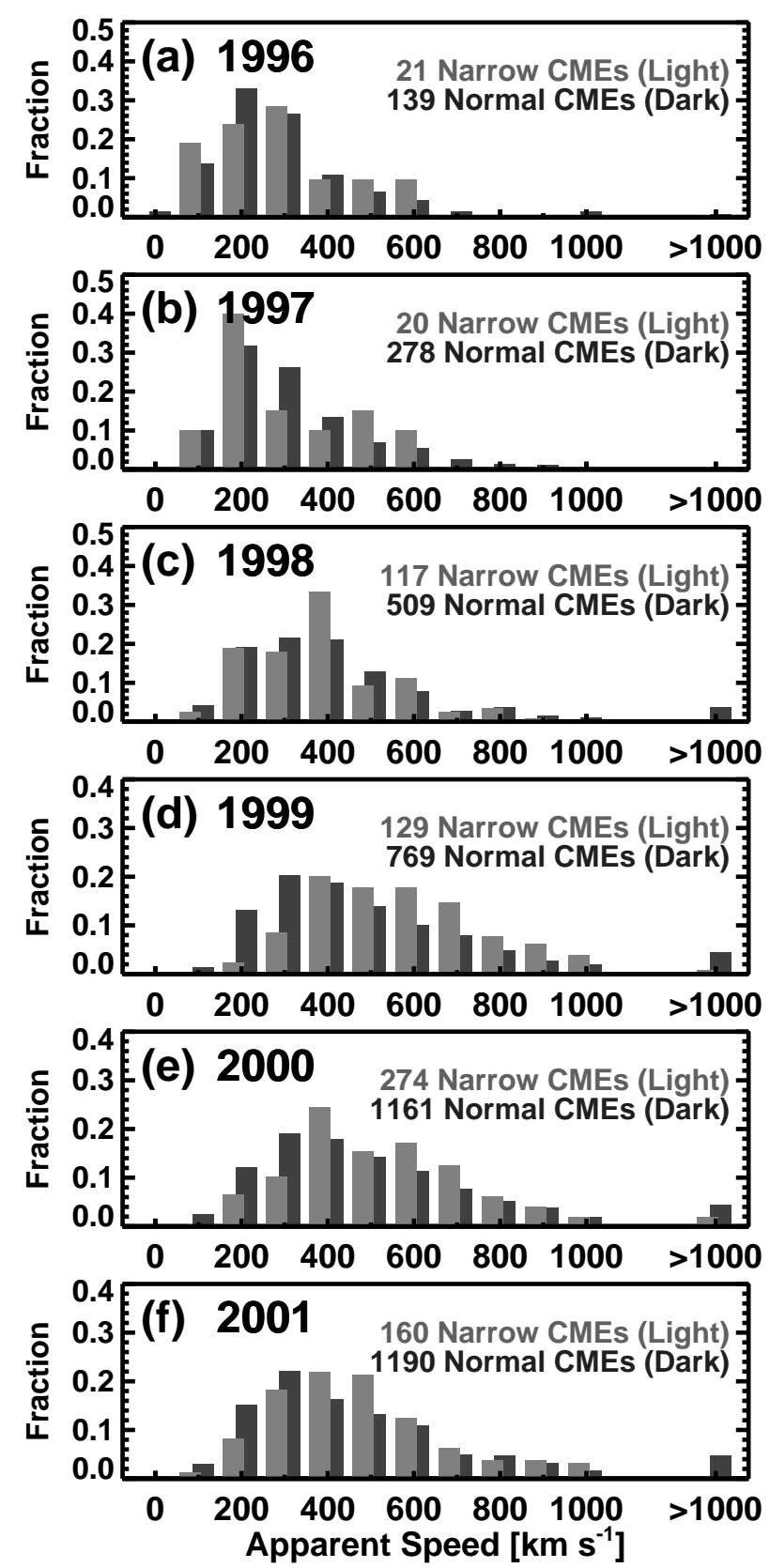

Fig. 2. Speed distribution for narrow (light-shaded) and normal (dark-shaded) CMEs from 1996 to 2001.

\section{LATITUDE DISTRIBUTION}

The CME location (central PA) was defined as the mid angle between two side edges. To investigate CME locations easily, we converted the CME location to the apparent heliographic latitude (see Gopalswamy et al. 2003). Figure 3 shows distributions of apparent latitude from 1996 to 2001 for narrow (light bars) and normal (dark bars) CMEs. During solar minimum, almost all CMEs occurred around the equator. The difference between narrow and normal CMEs was not clear because the number of narrow CMEs was too small. In 1998, the distributions for both narrow and normal CMEs became wider. During solar maximum (1999-2000), narrow CMEs appeared at all latitudes, similar to normal CMEs. A peak in figure 3e results from repetitive narrow CMEs at the same location. Six narrow CMEs occurred at PA of $70^{\circ}$ from November 3 to 6,2000 . The distributions for both narrow and normal CMEs were consistent with the distribution of streamers. No significant difference was found between narrow and normal CMEs in the latitude distribution. 


\section{SUMMARY DISCUSSION}

We investigated 806 narrow (width $<20^{\circ}$ ) CMEs to compare with 4311 normal (width $>20^{\circ}$ ) CMEs and found the following. The population of narrow CMEs increased towards solar maximum. Narrow CMEs were ejected approximately from the equatorial region during solar minimum, while during solar maximum narrow CMEs originated from all latitudes (similar to normal CMEs). No significant difference was found in the latitude distribution. Average speeds of narrow CMEs increased towards solar maximum from $300 \mathrm{~km} \mathrm{~s}^{-1}$ to 550 $\mathrm{km} \mathrm{s}^{-1}$. During solar maximum, the median speed of narrow CMEs was greater than that of normal CMEs, while the maximum speed of narrow CMEs was much smaller than that of normal CMEs.

Wang et al. (1998) examined the low-corona counterparts of 27 narrow CMEs and found EIT jets in all cases. Their jet-like CMEs have angular widths of $\sim 3^{\circ}-7^{\circ}$, the leading edge speed of $400-$ $1100 \mathrm{~km} \mathrm{~s}^{-1}$, and bulk flow speed of $\sim 250 \mathrm{~km} \mathrm{~s}^{-1}$. The speed of leading edge is consistent with our results. Shimojo and Shibata (2000) investigated X-ray jets observed by Yohkoh satellite. The speed of X-ray jets ranges from $10-1000 \mathrm{~km} \mathrm{~s}^{-1}$ similar to that of the narrow CMEs. However, the average speed of X-ray jets is only $200 \mathrm{~km} \mathrm{~s}^{-1}$, much lower than that of narrow CMEs. Note that Shimojo and Shibata concluded that the origin of X-ray Jet is evaporation flow results from flare heating due to magnetic reconnection.
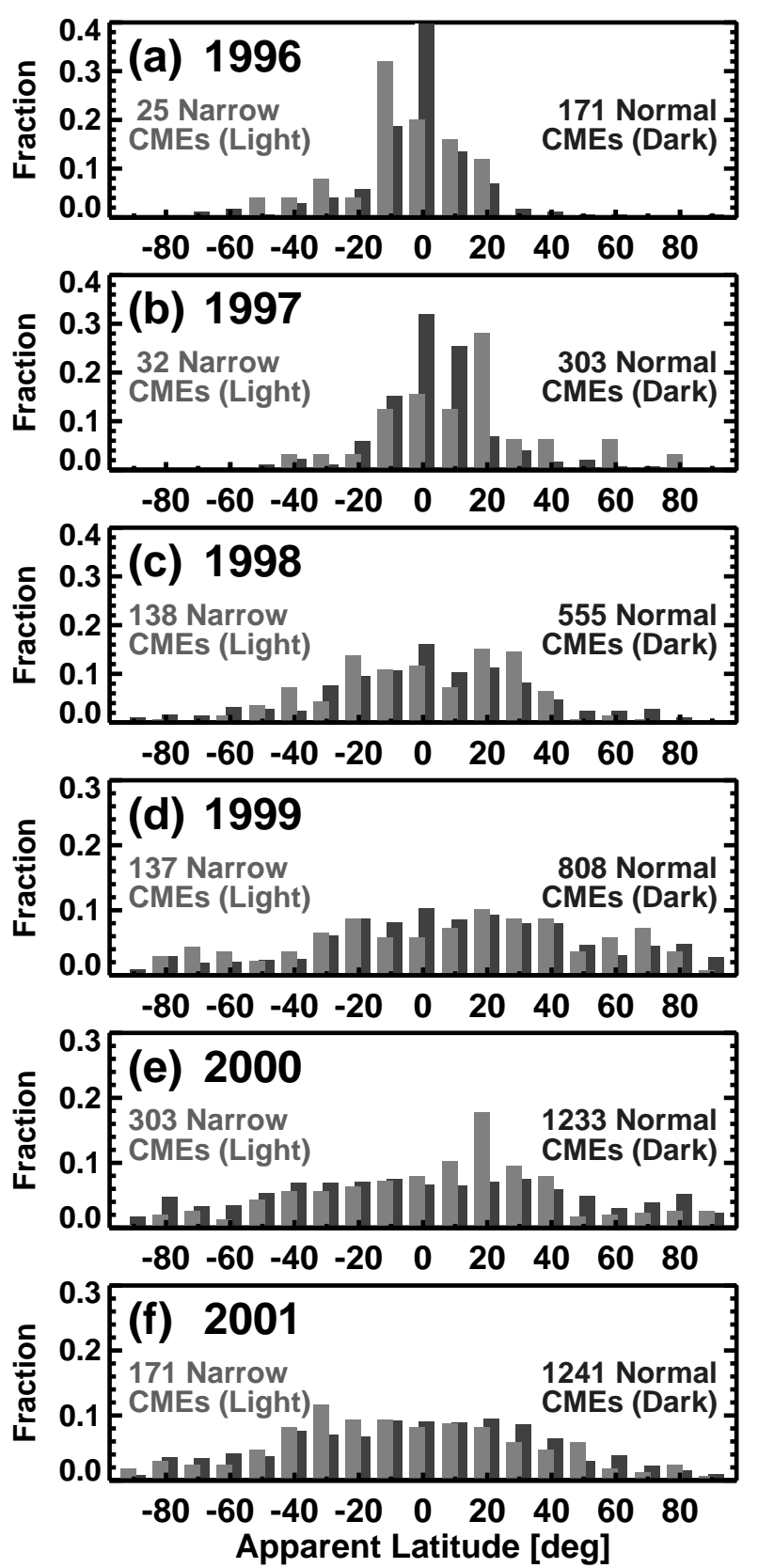

Fig. 3. Latitude distribution for narrow (light-shaded) and normal (dark-shaded) CMEs from 1996 to 2001.

\section{ACKNOWLEDGMENTS}

Authors would like to thank to referees for fruitful comments. The SOHO/LASCO data used here are produced by a consortium of the Naval Research Laboratory (USA), Max-Planck-Institut fuer Aeronomie (Germany)), Laboratoire d'Astronomie (France), and the University of Birmingham (UK). SOHO is a project of international cooperation between ESA and NASA. The catalog is developed in cooperation with the Naval Research Laboratory and the Solar Data Analysis Center (SDAC) at the Goddard Space Flight Center. Part of this effort was also supported by the Air Force Office of Scientific Research (AFOSR). 


\section{REFERENCES}

Gilbert, H. R., E. C. Serex, T. E. Holzer, R. M. MacQueen, and P. S. McIntosh, Narrow Coronal Mass Ejections, Astrophys. J., 550, 1093-1101, 2001.

Gopalswamy, N., A. Lara, R. P. Lepping, M. L. Kaiser, D. Berdichevsky, and O. C. St. Cyr, Interplanetary Acceleration of Coronal Mass Ejections, Geophys. Res. Letters, 27, 145-148, 2000.

Gopalswamy, N., S. Yashiro, G. Michałek, M. L. Kaiser, R. A. Howard, D. V. Reames, R. Leske, and T. von Rosenvinge, Interacting Coronal Mass Ejections and Solar Energetic Particles, Astrophys. J., 572, L103-L107, 2002

Gopalswamy, N., M. Shimojo, W. Lu, S. Yashiro, K. Shibasaki, and R. A. Howard, Prominence Eruptions and Coronal Mass Ejection: A Statistical Study Using Microwave Observations, Astrophys. J., 586, 562-578, 2003

Kahler, S. W., D. V. Reames, and N. R. Sheeley Jr., Coronal Mass Ejections Associated with Impulsive Solar Energetic Particle Events, Astrophys. J., 562, 558-565, 2001.

Shimojo, M. and K. Shibata, Physical Parameters of Solar X-Ray Jets, Astrophys. J., 542, 1100-1108, 2000.

St. Cyr, O. C., R. A. Howard, N. R. Sheeley Jr., S. P. Plunkett, D. J. Michels, S. E. Paswaters, M. J. Koomen, G. M. Simnett, B. J. Thompson, J. B. Gurman, R. Schwenn, D. F. Webb, E. Hildner, and P. L. Lamy, Properties of coronal mass ejections: SOHO LASCO observations from January 1996 to June 1998, J. Geophys. Res., 105, 18,169-18,185, 2000.

Webb, D. F., E. W. Cliver, N. U. Crooker, O. C. St. Cyr, B. J. Thompson, Relationship of halo coronal mass ejections, magnetic clouds, and magnetic storms, J. Geophys. Res., 105, 7491-7508, 2000.

Wang ,Y.-M., N. R. Sheeley Jr., D. G. Socker, R. A. Howard, G. E. Brueckner, D. J. Michels, D. Moses, O. C. St. Cyr, A. Llebaria, J.-P. Delaboudiniere, Observations of Correlated White-Light and ExtremeUltraviolet Jets from Polar Coronal Holes Astrophys. J., 508, 899-907, 1998.

Wang ,Y.-M. and N. R. Sheeley Jr., Coronal White-Light Jets near Sunspot Maximum, Astrophys. J., 575, $542-552,2002$.

E-mail address of S. Yashiro: yashiro@cdaw.gsfc.nasa.gov

Manuscript recieved 04 December, 2002; revised 10 March, 2003; accepted 10 March, 2003 